\title{
3applicability of The LOW-TEMPERATURE IGNITION METHgD FOR DETERMINING CARBON IN VARIOUS ALKALI METALS
}

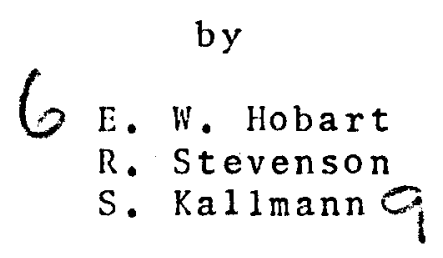

National Aeronautics and Space Administration

$$
\begin{aligned}
& \text { Goctober } 21,196610 \\
& \text { Contract No. NASW-1192-29 } \\
& \text { I Ledoux \& Company } \\
& \text { Teaneck, N.J. } 3
\end{aligned}
$$

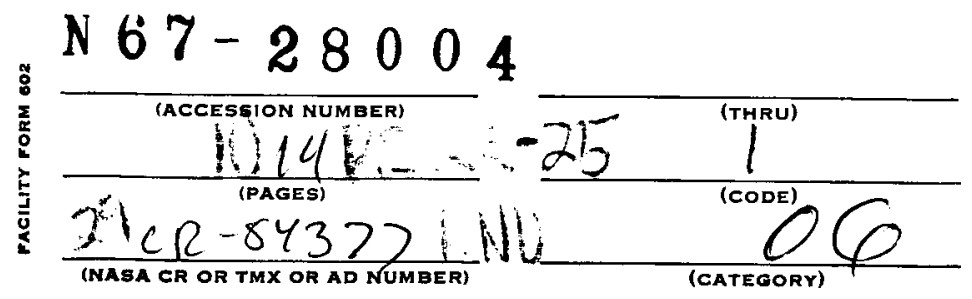


APPLICABILITY OF THE LOW-TEMPERATURE IGNITION METHOD FOR DETERMINING CARBON IN VARIOUS ALKALI METALS 


\section{ABSTRACT}

Kallmann and Liu's low temperature ignition method for carbon in sodium has been extended to the other alkali metals. Good recoveries of carbon, added as sucrose, potassium acid phthalate, carbon black and alkali carbonate, have been achieved for the metals: NaK alloy, potassium, rubidium and cesium. Lithium, however, is not amenable to the method. Gas Chromatographic measurement of carbon dioxide has extended the sensitivity of the method to the one prm carbon range. 
INTRODUCTION

In 1964, Kallmann and Liu published a novel method for determining carbon and sodium carbonate in sodium metal (1). The method involved oxidation of sodium samples to sodium oxide at a relatively low temperature in an oxygen-argon atmosphere. During the process, any carbon in the samnle was converted to sodium carbonate, a stable compound at temperatures below about $700{ }^{\circ} \mathrm{C}$. Carbon dioxide was released from the sodium oxide-sodium carbonate mixture by treatment with sulfuric acid and was measured by noting the change of conductance of a dilute barium hydroxide solution on absorption of the carbon dioxide. Carbonate in sodium was determined by decomposing the sample to sodium hydroxide in a stream of moist argon followed by acidification to release carbon dioxide. The objectives of the present study were (1) to increase the sensitivity of the method and (2) to establish its applicability to the other alkali metals. 
APPARATUS:

The ignition apparatus is shown in Figures 1 and 2 . The trapping system, depicted in Figure 3 , consists of a drying tube filled with magnesium perchlorate, a gas sampling valve, (F \& M Model GV-11), an injection sentum fashioned from a "T" tube fitting and a $1 / 4$ " diameter $x$ 1/16" thick disc of neoprene, a molecular-sieve packed trap (LECO part Number 589-613), a 100 ampere, 1.4 volt transformer (LECO 589-540), and a time switch ( $\theta-60$ second darkroom time switch is suitable). The chromatograph is an F \& M Model 700 equipped with Type WX filament thermal conductivity detector and a Sargent integrating recorder Model SR-35. The operating conditions of the chromatograph are noted in Table I.

Table I. Operating Conditions for Gas Chromatograph

Column $18^{\prime \prime} \times 1 / 4^{\prime}$ Silica Gel
Column Temp. $-130^{\circ}$
Detector Temn. $-90^{\circ}$
Detector Current $-100 \mathrm{ma}$
Carrier gas - helium, $60 \mathrm{cc}$ min.


MATERIALS:

Potassium and rubidium were supplied by NASA in stainless steel tubes. Samples of potassium were extruded while rubidium samples were obtained by cutting pieces of the tubing. Because of the low melting point of rubidium $\left(38^{\circ}\right)$, handing of the samples proved to be difficult. Additional $5 \mathrm{~g}$ samples of rubidium, potassium and NaK were obtained from Mine Safety Appliances Research in sealed glass ampoules. Fifteen gram samples of cesium in glass ampoules were provided by Mr. C. D. Houston of the Air Force Materials Laboratory, Wright-Patterson AFB.

PROCEDURE:

After loading the sample into the reaction tube under argon, the apparatus is assembled with the exit end of the reaction tube open to the atmosphere. While maintaining a flow of argon through the reaction chamber, the chamber is heated in the area of the sample until the alkali metal melts and flows into the zirconium crucible. After cooling the apparatus, argon is partially replaced by oxygen which enters the gas stream through a "Y" connection. The reaction tube is heated gently until the alkali metal begins to ignite. The oxygen/argon ratio is adjusted continuously to allow the sample to continue to burn at the lowest possible temperature. As the reaction subsides, the proportion of oxygen is increased until the sample is finally ignited at about $600^{\circ}$ in pure oxygen. 
When all reaction has ceased, the chamber is allowed to cool. Freshly boiled cold water is placed in a gas washing bottle and further purified by passing argon through it for a few minutes. The gas washing bottle is introduced into the assembly and the stopcocks are turned so that argon will push the water into the reaction tube. The latter is now connected to a sulfuric acid bubbler and thence to the trapping system. A second gas washing

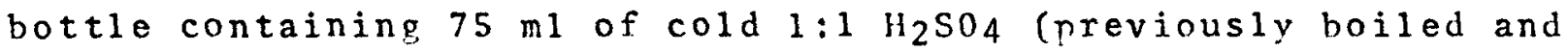
further purified by passing argon through it) is now positioned in the assembly. The gas sampling valve of the chromatograph is placed in the Inject position and the trap is heated. After about five minutes, the valve is switched to the Collect position and stopcocks are manipulated so that argon will nush the acid into the reaction tube.

After twenty minutes of collecting $\mathrm{CO}_{2}$, the gas sampling valve is switched to the Inject nostion. The chromatograph bridge is balanced with minimum attenuation. When a stable base-1ine is obtained, the attenuator is set to the estimated range for the sample and then the time switch is triggered to heat the molecular sieve trap for 30 seconds. The carbon dioxide peak, which appears in 120 seconds, is measured and converted to micrograms of carbon by use of a calibration factor. Calibration is accomplished either by injecting known volumes of $\mathrm{CO}_{2}$ into the collection trap or by introducing standardized sodium carbonate solutions into the re- 
action vessel and carrying out the procedure starting with the acid addition.

\section{RESULTS AND DISCUSSION}

\section{SENSITIVITY:}

In the original work, carbon dioxide was measured conductometrically. This technique is suitable for determining down to about 10 micrograms of carbon. The gas chromatographic method described in this report is suitable for the determination of as little as 1 microgram of carbon. About ten fold additional chromatographic sensitivity can be obtained by increasing the bridge current to $230 \mathrm{ma}$. However, the blank variability of about one-half microgram makes this futile. The lower current of 100 ma was used to extend the life of the detector filaments while maintaining the ability to detect one-half to one microgram of carbon over the blank of $2 \pm 0.5 \mathrm{micrograms.}$ Since several gram samples are readily accommodated when using this method, carbon in the range of 0.1 to 1 pnm should be determinable. The nurest alkali available to us was cesium, a fifteen gram sample of which was analyzed to contain 1.7 ppm of carbon, all present in the form of carbonate.

CARBON IN LITHIUM:

Of all the alkali metals, lithium proved to be the most difficult to analyze by the low-temperature ignition method. Samples 
of lithium will not ignite, even in pure oxygen, unless moisture is added to the atmosphere. Using a water filled gas washing bottle to moisten the argon and oxygen atmosphere, ignition could be started with about $10 \%$ oxygen. The combustion is difficult to control, however, and if excessive temperatures are attained some carbon dioxide is lost. The difficulties are demonstrated by the results shown in Table II. Also shown in Table II are the results for determining 1 ithium carbonate in lithium. In this case, lithium is the easiest of all of the alkali metals to analyze. Samples react smoothly with water in the reaction vessel and carbon dioxide is subsequently released by acidification. In the case of lithium, carbon is best determined by acetylene evolution for carbide carbon and by this method for carbonate. Evidence for the validity of acetylene measurement as a means of determining carbon in 1 ithium has been reported (2). Gilbert,Meyer and white nublished a method for determining carbon in lithium by acetylene evolution (3).

\section{Table II. Carbon in Lithium}

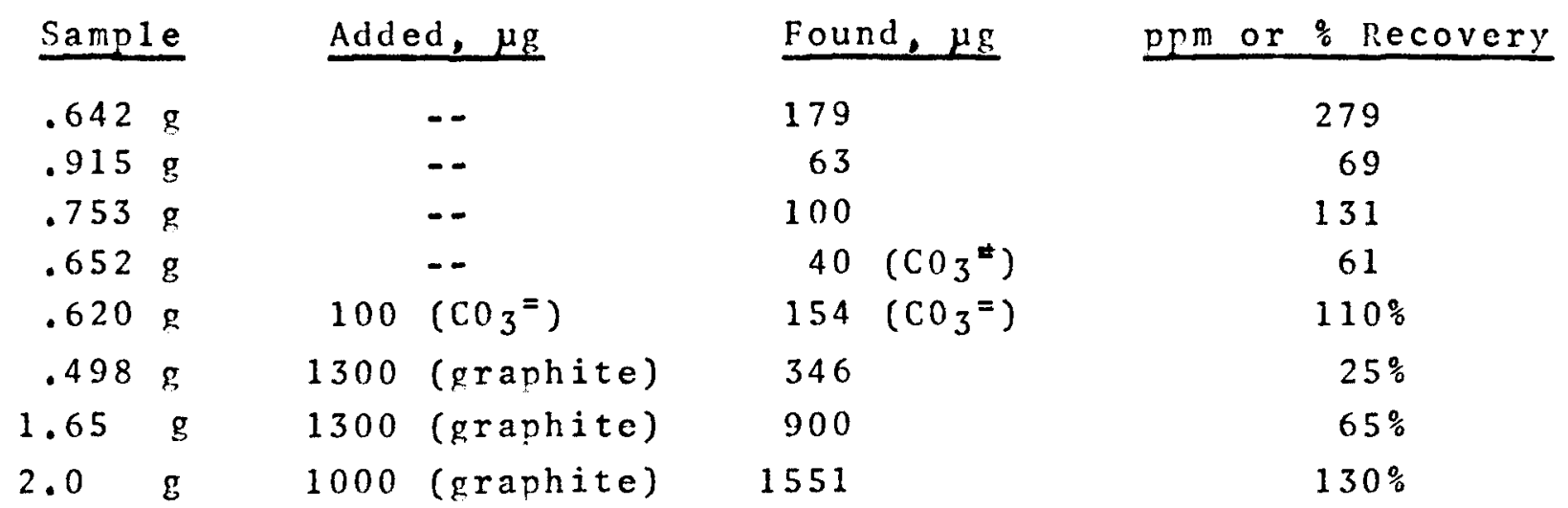


CARBON IN POTASSIUM:

Potassium samples are smoothly ignited in the regular manner. Carbonate carbon is determined by pasing moist argon over the heated sample until the sample is converted to potassium hydroxide followed by the usual dilution, acidification and transfer of carbon dioxide to the chromatograph. Results presented in Table III demonstrate the utility of the method for analyzing potassium.

Table III. Carbon in Potassium

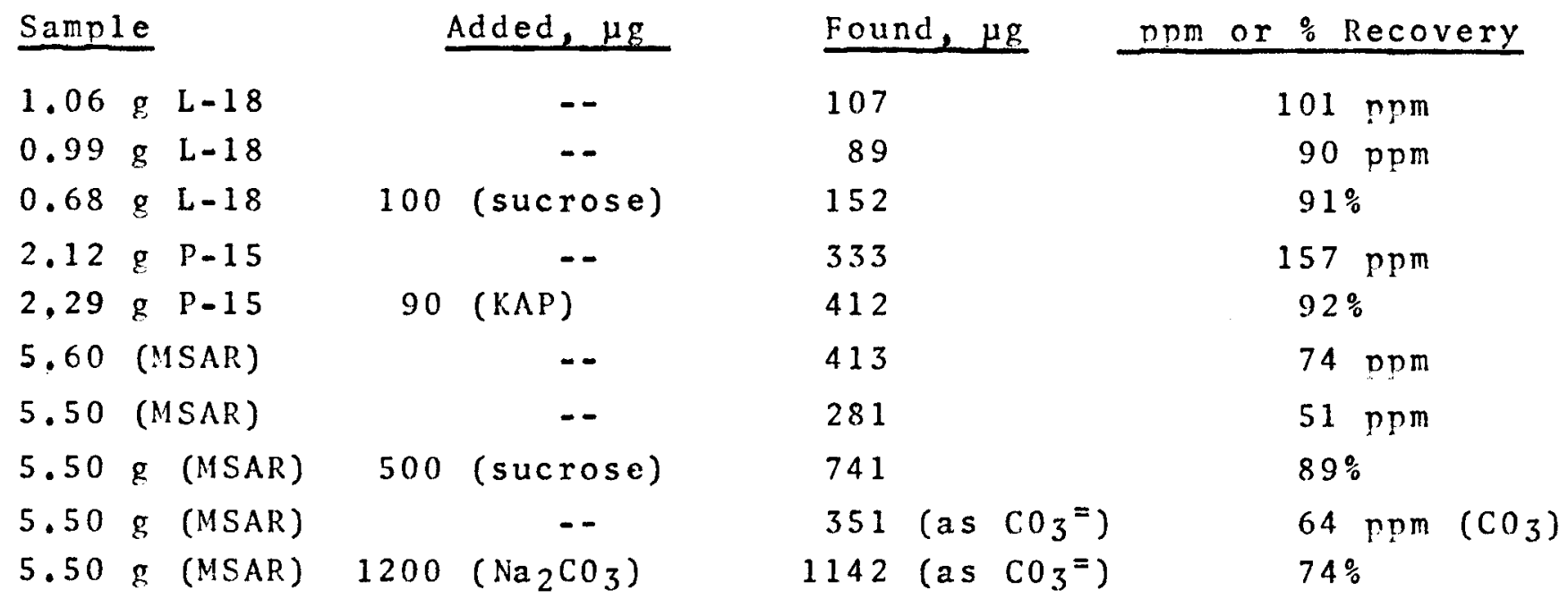

CARBON IN CESIUM:

Cesium samples burn well in the standard procedure. Reasonable results of recovery experiments, shown in Table IV, demonstrate that the method is applicable to analysis of cesium. Carbonate in cesium is determined by heating the cesium in moist argon to form cesium hydroxide followed by the usual diluting, acidification and 
collection of carbon dioxide. Because of the high reactivity of cesium and of its tendency to form a protective coating of hydroxide, the hydrolysis process is rather difficult to nerform. Sufficient heat must be applied to cause the hydroxide to react with free cesium and yet excessive heat must be avoided to prevent decomposition of the carbonate. However, the results in Table IV show that both total carbon and carbonate carbon can be determined. Furthermore, they demonstrate the excellent sensitivity of the method when using gas chromatographic measurement of the carbon dioxide.

Table IV. Carbon in Cesium

$\begin{array}{lccc}\text { Sample } & \text { Added, ug } & \text { Found, ug } & \text { prm or } \% \text { Recovery } \\ 15.0 \mathrm{~g} & -- & 25.7 & 1.7 \mathrm{ppm} \\ 15.0 \mathrm{~g} & 100 \text { (sucrose) } & 116 & 93 \% \\ 15.0 \mathrm{~g} & 500 \text { (sucrose) } & 803 & 128 \% \\ 15.0 \mathrm{~g} & -- & 27\left(\mathrm{CO}_{3}=\right) & 1.8 \mathrm{ppm} \\ 15.0 \mathrm{~g} & 100\left(\mathrm{Na}_{2} \mathrm{CO}_{3}\right) & 99.5 & 79 \%\end{array}$

CARBON IN RUBIUIUN:

The analysis of rubidium samples proceeded very similarly to that of cesium using the standard procedure. Results shown in Table $V$ demonstrate the applicability of the method. 
Table V. Carbon in Rubidium

\begin{tabular}{|c|c|c|c|c|c|c|}
\hline Samp & & & dded, $\mu g$ & Found, ug & nnm or & Recovery \\
\hline 7.15 & $g$ & & -- & 209 & & $28 \mathrm{ppm}$ \\
\hline 7.5 & $g$ & & $\ldots$ & 162 & & $22 \mathrm{ppm}$ \\
\hline 6.7 & $g$ & 100 & (sucrose) & 202 & & $73 \%$ \\
\hline 7.0 & $g$ & 500 & (sucrose) & 602 & & $90 \%$ \\
\hline 7.1 & $g$ & & $\ldots$ & $226\left(\mathrm{CO}_{3}=\right)$ & & $32 \mathrm{prm}$ \\
\hline 6.0 & $g$ & 600 & $\left(\mathrm{CO}_{3}=\right)$ & $650\left(\mathrm{CO}_{3}=\right)$ & & $84 \%$ \\
\hline
\end{tabular}

CARBON IN SODIUM-POTASSIUM ALLOY:

Sodium-Potassium alloy (44-56 NaK) was analyzed by the standard method. No particular difficulties were encountered. Pesults are presented in Table VI.

Table VI. Carbon in NaK

\begin{tabular}{|c|c|c|c|}
\hline Sample & Added, $\mu g$ & Found, $\mu g$ & nnm or $\%$ Recovery \\
\hline $6.5 \mathrm{r}$ & -- & 109 & $17 \mathrm{ppm}$ \\
\hline $5.25 \mathrm{~g}$ & 500 (sucrose) & 549 & $91 \%$ \\
\hline 5.95 & 500 (sucrose) & 506 & $85 \%$ \\
\hline
\end{tabular}

SAMPLE HANDLING:

Ilandling of the alkali metals requires careful exclusion of atmospheric contamination. Since we do not have high quality dry boxes for handling, these metals, we avoided the problem by concentrating our efforts on analvzing samples obtained in glass ampoules. Extrusion from steel tubes was used to sample notassium but could not be applied to rubidium or cesium because of their low melting points. Lithium samples were received under mineral oil. They 
were freed of oil by washing twice with hexane, then with ethanol, and finally with water. The washed samples vere dropped immodiately into the reaction tube. The ultimate limits of detection of carbon in alkali metals by this method are established by the degree of contamination of samples.

\section{CONCLUSION:}

The low temperature ignition method for determining carbon in sodium is applicable to all of the alkali metals with the excention of lithium. In the case of lithium, the difficulty in controlling the rate of ignition coupled with the relative instability of lithium carbonate nrecludes satisfactory determinations of total carbon. Carbonate, on the other hand, is readily determined in lithium by this method. In other work, it has been shown that carbon is present in lithium as a carbide which quantitatively yields acetylene on hydrolysis. Thus, lithium samples can be hydrolyzed and acetylene determined gas chromatographically or photometrically followed by acidification of the solution to release carbon dioxide for determination of the carbonate. 


\section{LITERATURE CITED}

1. Kallmann, S. ELiu, R., Anal. Chem. 36, 590 (1964)

2. Hobart, E.W. \& Bjork, R.G., Manuscript A722 submitted to Ana l. Chem.

3. Gilbert, T. W., Meyer, A.S. and White, J. C., Anal. Chem. 29, 1627 (1957) 


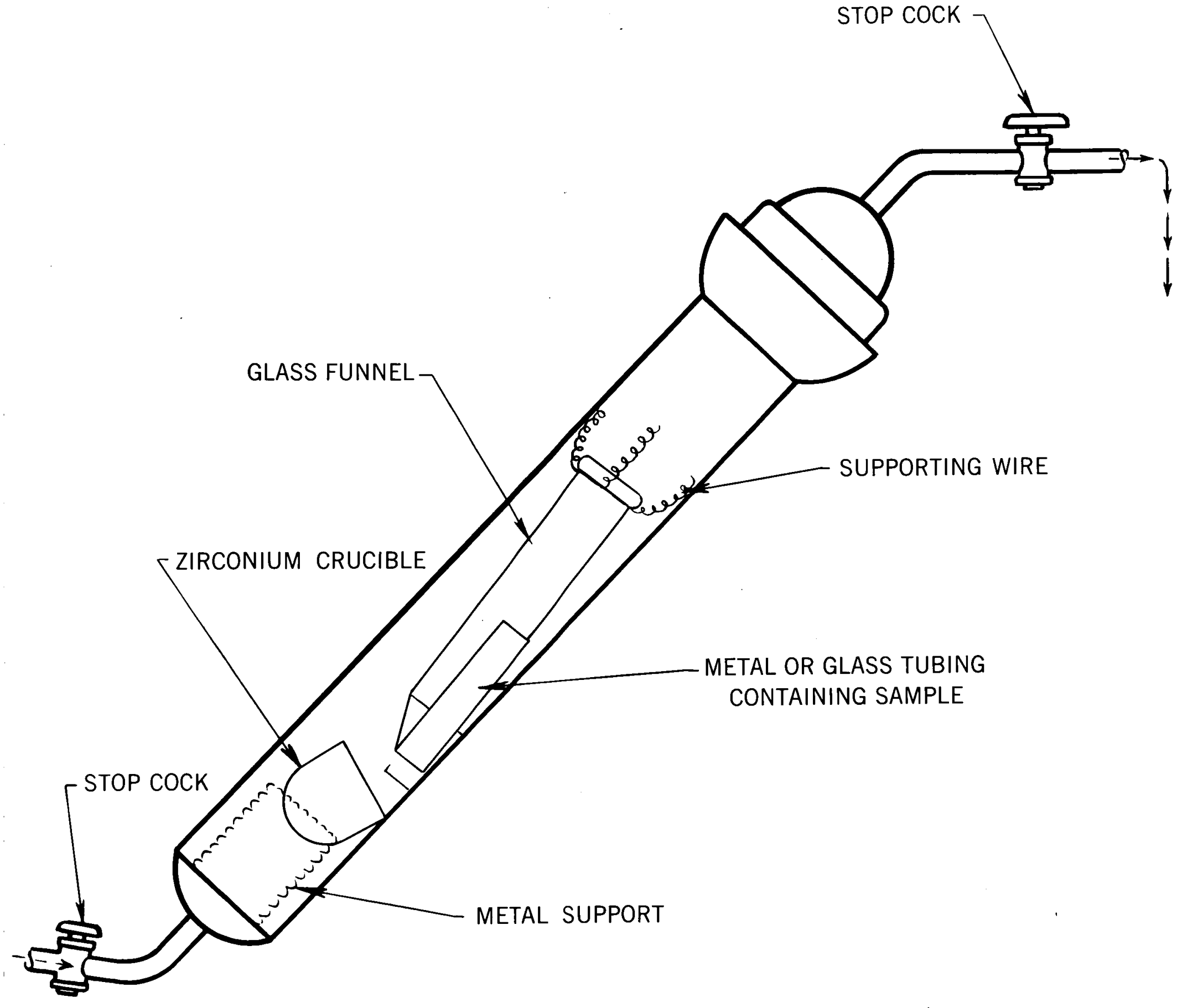

FIGURE 1 Reaction Tube 


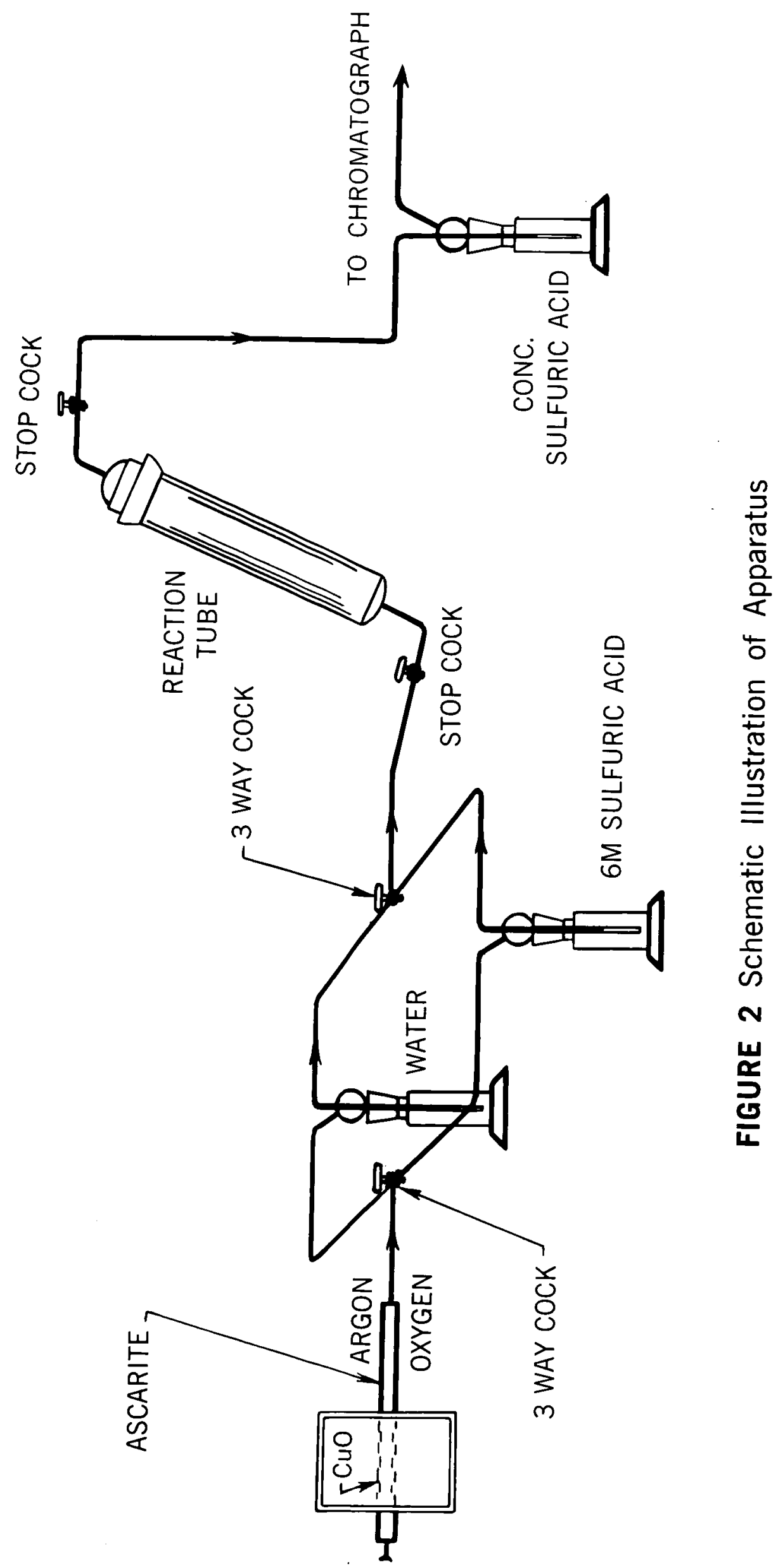




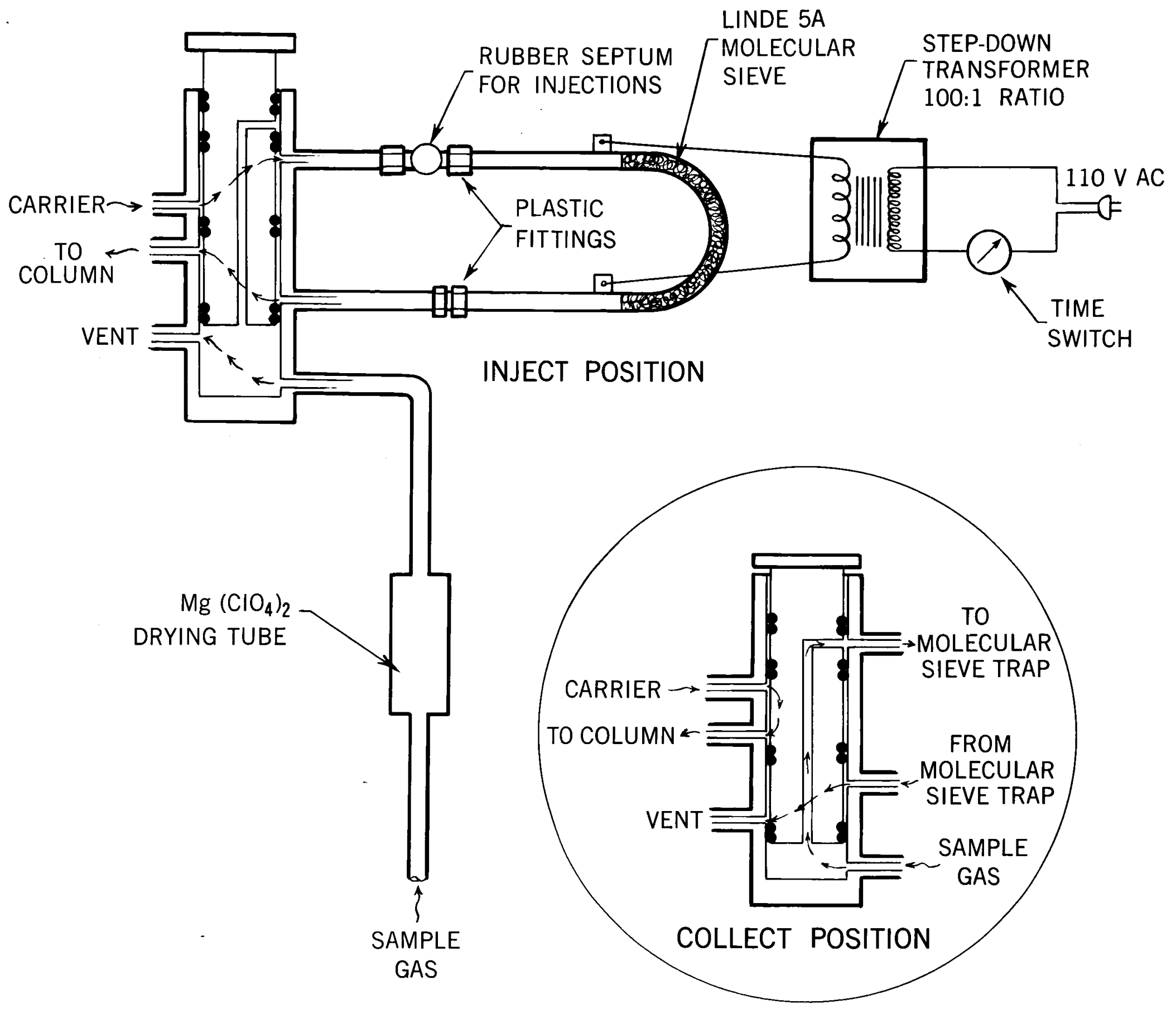

FIGURE $3 \mathrm{CO}_{2}$ Trapping System 